\title{
E-learning en la enseñanza del alemán como lengua extranjera
}

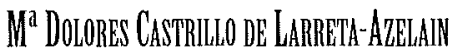

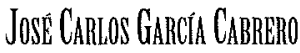 \\ GerMar Ruprériz \\ UNED, Madrid.
}

Introducción En un sentido amplio, podríamos definir -learning como el sistema de enseñanza / aprendizaje que utiliza las tecnologías proporcionadas por Internet (Rosenberg 2001:28). Sin embargo, este término se utiliza muchas veces para hacer referencia a modelos educativos diferentes, en los que, en general, coexisten integrados en un sistema mixto las sesiones o tutorías presenciales voluntarias u obligatorias, el material didáctico impreso, la tutorización telemática, etc.

De esta forma, se suele denominar e-learning tanto a los modelos que utilizan sólo Internet para la distribución del material didáctico y para llevar a cabo la acción tutorial - lo que podríamos denominar e-learning puro - como a las iniciativas que utilizan la red como apoyo a la enseñanza presencial y también a las que la usan de ayuda a la enseñanza a distancia más tradicional'. Parece evidente que, según el paradigma educativo correspondiente, las debilidades y las fortalezas cambian considerablemente, por lo que resulta complicado extraer conclusiones extrapolables de unos modelos a otros.

En el ámbito que nos ocupa, la enseñanza de las lenguas modernas, en general, y del alemán en particular, son poco frecuentes, por no decir prácticamente inexistentes, los sistemas que utilizan sólo e-learning, pues las dificultades técnicas para poner en práctica algunas habilidades como la expresión oral así lo desaconsejan. Los paradigmas actuales se basan en un sistema mixto, con mayor o menor presencia de las partes virtual y presencial según los casos, que se suelen englobar bajo la denominación blended-learning. Sintética y acertadamente, Rizek-Pfister (2003:146) definió este último término como «Mix von Fernund Präsenzunterricht». Sauter et al. (2004:15) desarrollan la definición de la siguiente manera:

«E-Learning-Ansätze sind insbesondere dann wirksam, wenn sie in das Konzept eines Lernarrangements eingebunden sind, welches neben computergestützten Elementen auch 'klassische' Lernformen [...] umfasst. Daher wird dieses System auch 'blended' genannt [...]. E-Learning ersetzt damit nicht klassis- che Lernformen, sondern ergänzt und bereichert sie.» Rossett et al. (2003) precisan un poco más:

"A blend is an integrated strategy for delivering on promises about learning and performance. Blending involves a planned combination of approaches, such as coaching by a supervisor; participation in an online class; breakfast with colleagues; competency descriptions; reading on the beach; reference to a manual; collegial relationships; and participation in seminars, workshops, and online communities».

Por estas razones, nos ha parecido natural y apropiada para su presentación en este artículo una división de la potencialidad del e-learning como apoyo a la enseñanza presencial, por un lado, y como ayuda en la docencia a distancia, por otro.

Estos modelos educativos mixtos o blended-learning surgieron para intentar lograr un uso más racional de la tecnología en el proceso educativo. Históricamente, cada vez que se produce un nuevo salto tecnológico (la aparición del CD-ROM o de la Web, por ejemplo) se suele generar un sentimiento de euforia por haber encontrado la solución a todos los males educativos, aunque, con el paso del tiempo, la mayoría de estas promesas se quedan en meros deseos imposibles de cumplir: parece ser que no es el medio sino el contenido lo que verdaderamente importa (Weller 2002:7). La experiencia histórica demuestra que, generalmente, los «nuevos medios» se integran con los ya disponibles, pero que éstos no desaparecen.

Sin embargo, sí es verdad que Internet es diferente frente a otras revoluciones tecnológicas y su impacto en la educación, como el CD-ROM, por ejemplo, pues es una tecnología «rompedora, no continuista" (Christensen 1997:xv) por rasgos como su impresionante aceptación social, las interfaces genéricas - el alumno usa el mismo programa, un explorador de páginas $W e b$, para estudiar o para su tiempo de ocio -, las capacidades interactivas, de personalización, de actualización continua, etc. (Weller 2002:10-16).

Además de las citadas anteriormente, entre las características más importantes es necesario destacar, sin duda, los cambios de 

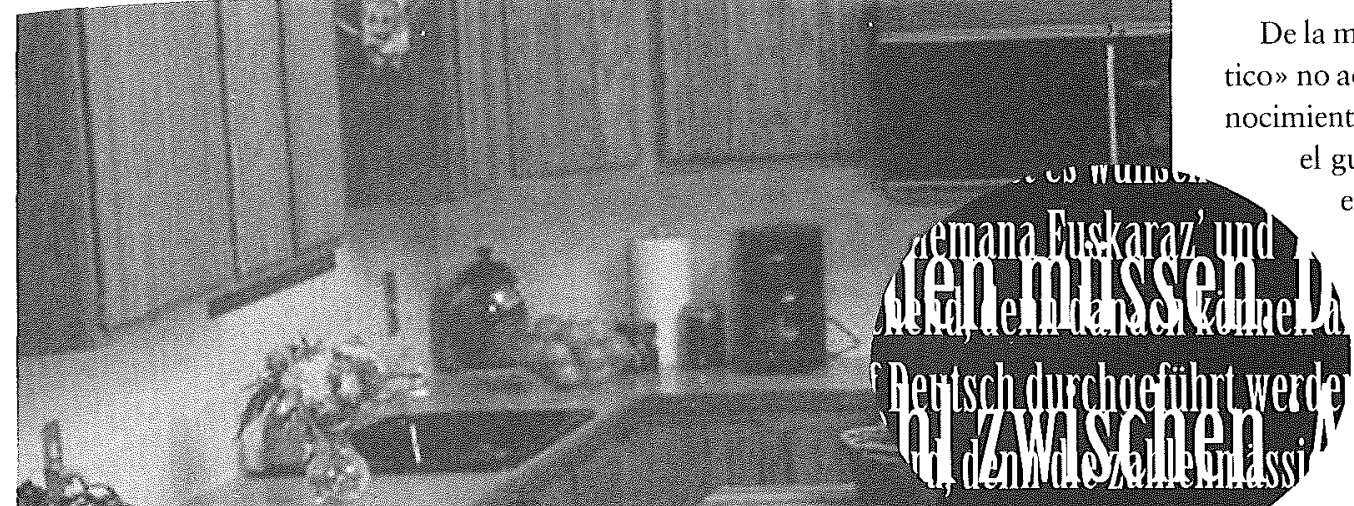

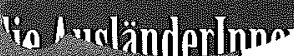

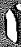

(1)

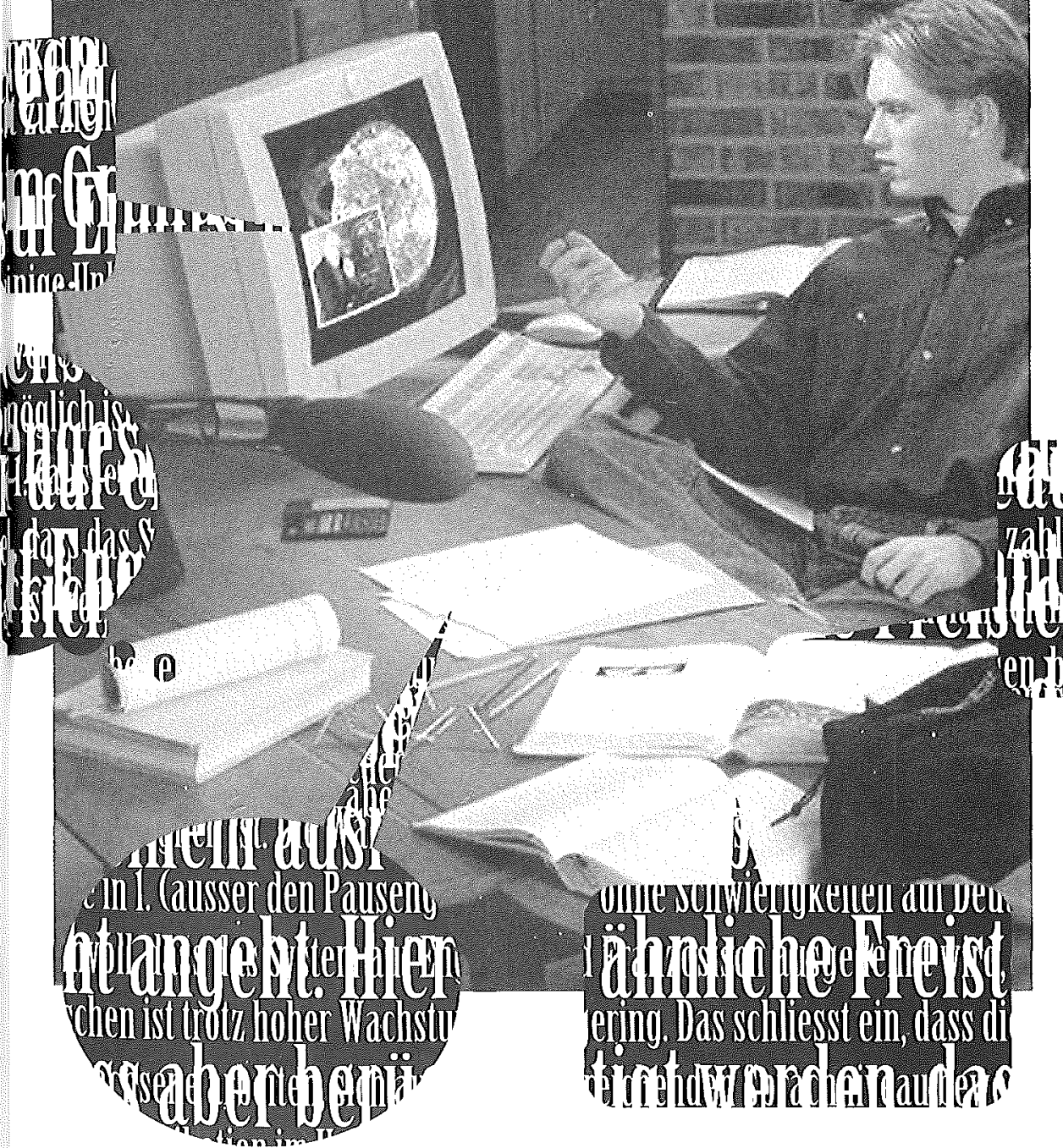
extranjera:

De la misma forma, el «profesor telemático" no actúa sólo como transmisor de conocimientos, sino que más bien pasa a ser el guía del alumno en su proceso de exploración y construcción de conocimiento. Weller (2002:77) afirma en este sentido:

"The educator's function is now first to create the learning opportunities and then to guide students through these, while allowing the students to be active in the learning process."

Los factores clave del éxito de la introducción de Internet en la práctica docente son, sin duda, la actitud de alumnos y profesores, su asimilación de estos nuevos papeles $y$, por supuesto, unos contenidos de calidad adaptados al medio. Como afirma Cruz Piñol (2002:11), refiriéndose en concreto al caso del español como lengua

«[...] ha hecho proliferar las Webs de enseñanza del español como lengua extranjera (ELE). Pero ocurre que estos materiales a veces resultarían más manejables y puede también más prácticos en un libro impreso, en una cinta de casete, en un vídeo o en un cederrón. Este hecho provoca el escepticismo - lógico y fundado-de muchos profesores de español que acuden a Internet en busca de algo supuestamente maravilloso y se encuentran con lo mismo que conocen desde hace años y, en ocasiones, con numerosas limitaciones añadidas".

\section{El E-learning LainclusióndeIn- en la enseñanza ternet en el pro- presencial del ceso de enseñan- za de lenguas ex- alemán como tranjeras engene- lengua extranjera ral, y del alemán en particular, es}

un hecho ampliamente documentado y que,

roles que se producen en este paradigma educativo, pues es el alumno quien estudia solo delante del ordenador, pasa de convertirse en un receptor - y, por tanto, pasivo en la mayoría de las ocasiones - de la clase magistral de la enseñanza presencial tradicional, a ser él quien directamente gestiona y se responsabiliza de su aprendizaje. según algunos autores como Steinig (2000:125-156), se debe al estado preciso en que se encontraba la discusión metodológicodidáctica en el campo de la enseñanza de lenguas en el momento en que surgieron las aplicaciones tecnológicas asociadas a Internet. En la actualidad podemos encontrar múltiples y variados recursos en este nuevo medio digital que pueden colaborar de 
manera significativa en el proceso de adquisición de una lengua extranjera, puesto que, como afirman autores como Borrmann y Gerdzen (2000:4):

«Das neue Medium belastet den Deutschunterricht nicht durch noch mehr Inhalt, sondern verändert, ja verbessert ihn vor allem in methodischer Hinsicht."

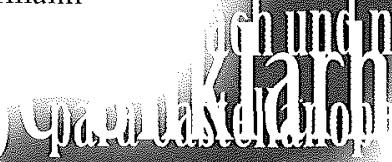

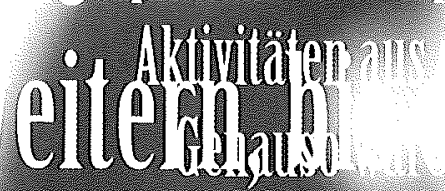

El entorno de aprendizaje virtual añade nuevas vías para el desarrollo del acto formativo, algunas de cuyas fases (Kolb, 1984) se desarrollan sin duda de manera más adecuada en este nuevo medio. En este apartado mostraremos ejemplos concretos de algunos recursos digitales válidos para su implantación en entornos presenciales de aprendizaje del alemán como lengua extranjera. La Web puede utilizarse como un recurso complementario a las clases presenciales con diferentes objetivos. Entre otros, Ko y Rossen (2001:14) mencionan la utilidad de este medio como fuente de información complementaria para el alumno tras las sesiones pre senciales, y también como un recurso adecuado para preparar las mismas. El procedimiento para integrar Internet en entornos presenciales es objeto de análisis por parte de muchos autores. Rosenberg (2001:119 ss.) apunta en este sentido:

"Hou shoulde-learning be used to supplement classroom leaming? There are many opportunities, especially for enrichment and follow-on learning. The Web can serve as a 'community wrapper' of sorts, keeping those in the knowledge community in touch with each other and with the content, either after the formal learning is concluded or between learning events. [...] E-Learning tends to be short, targeted, taskdriven and episodic, while classroom learning tends to be longer, less-well targeted, and programmatic."

Más recientemente, Breilmann, Grunow y Schopen (2003:7) afirman a este respecto:

«Ein medienintegrativer Deutschunterricht eröffnet zahlreiche neue Lern- und Kommunikationsmöglichkeiten: Schülerinnen und Schüler recherchieren nicht mehr allein in der Bibliothek, sondern nutzen auch kompetent das Internet. Sie verfassen und überarbeiten Texte am Computer, sic lesen und bewerten Webseiten, mit Lernsoftware können sie individualisiert und selbstständig üben. [...] besonders die neuen Kommunikationsformen wie Chat und SMS bieten Anlässe zur Sprachreflexion."

Una posible clasificación de los recursos en la Web para el aprendizaje del alemán contempla tres grandes grupos:

1 Índices o catálogos de recursos.

1 Páginas con diseño didáctico.

1 Páginas sin diseño didáctico.

El primer grupo, índices o catálogos, hace referencia a páginas que incluyen selecciones de enlaces útiles pa- ra la enseñanza/aprendizaje del alemán de tipología variada (directorios, catálogos de enlaces, listas comentadas, etc.). Este tipo de recursos es muy útil para una documentación previa o también posterior a la clase presencial, lo que, Döring (1998), por ejemplo, incluye en su clasificación en el apartado «Dozentenzentrierter Präsenzunterricht mit dem Internet» y describe como «Vor- und Nachbereitung mit Netzressourcen (z.B. Suche nach Quellen ... )». Este tipo de recursos es, sin duda, también muy adecuado para que los alumnos realicen búsquedas y resuelvan tareas dentro de unos parámetros definidos, sin el peligro de desviarse del objetivo con la consecuente pérdida de tiempo y demás "peligros" inherentes a las búsquedas sin filtro ni restricciones por Internet. Destacamos aquí las tres direcciones siguientes 'Linksammlungen':

1 Institut fiir Deutsche Sprache (IDS) <http://www.ids-mannheim.de/service \#Links>: se trata de uno de los catálogos de enlaces sobre alemán como lengua extranjera mejor estructurados y más extensos.

1 Forum Deutsch als Fremdsprache (IKK) $<$ http://www.deutsch-als-fremdsprache.de/daf-links/linksammlung>: esta colección de enlaces es una de las más completas clasificaciones de recursos de alemán como lengua extranjera e incluye categorías como foros, listas de distribución, herramientas de autor; tutoriales, exámenes oficiales, etc.

1 Germanistik.org

<http://www.uned.es/germanistik >: un catálogo que recoge más de 2.000 enlaces referidos al alemán como lengua extranjera y a la Germanística comentados y clasificados en torno a siete categorías.

El segundo grupo, páginas con diseño didáctico, incluye páginas diseñadas específicamente para el aprendizaje del alemán como lengua extranjera con metodología propia y objetivos didácticos. En este apartado incluimos, por una parte, páginas con ejercicios sobre léxico, de contenido morfosintáctico, de comprensión lectora, etc., por otra parte hacemos referencia también a páginas que inclu yen herramientas de comunicación orientadas al aprendiza je del alemán. La inclusión de las primeras como complemen to a la clase presencial persigue objetivos claros como el refuerzo del aprendizaje, el fomento de la autonomía del alumno y la posibilidad de autoevaluación. Asimismo no debemos despreciar el aumento de la motivación que supone, para una gran mayoría de alumnos, la inclusión de las nuevas tecnologías en su proceso de aprendizaje. Por otra parte, la utilización de las

herramientas de comunicación como un sistema complementario a la clase presencial, además de las ventajas más re ferenciadas por todos los investigadores y que hacen 
alusión a que el proceso formativo se desliga de los parámetros clásicos espacio-temporales, persigue objetivos muy definidos y claros como el fomento de la conciencia de grupo, característica fundamental que debe reunir todo proceso formativo según investigadores como Bender (2003:3), quien afirma: «Feeling included in a group is an important factor for encouraging the true potential for learning to take place».

En esta categoría podemos incluir las siguientes direcciones:

1 Web Projekte del Goethe Institut

<http://www.goethe.de/dll/mat/auf/prj/deindex.htm>: incluye material y cursos para la práctica del alemán. La muestra ofrece asimismo ejercicios tipo WebQuest que pueden utilizarse para el trabajo cooperativo entre los alumnos. Este espacio recoge, además, enlaces y proyectos en torno a chats, foros, y el uso correo electrónico.

1 Gerberonline

$<$ http://www.gerberonline/webquests/bw/prozess.html>: página de S. Gerber, que incluye mucho material para WebQuest, p.e. "Online-Bewerbung"

1 Hueber Online

<http://www.hueber:de/elearning>: se trata de un curso básico de alemán que consta de diez unidades con más de cien ejercicios interactivos de tipología variada, destacando los ejercicios que fomentan el trabajo cooperativo y los ejercicios tutorizados.

1 Interaktive Übungen (TU Darmstadt) <http://www.spz.tudarmstadt.de/imss/Deutsch/M2/index.html> contiene una batería de ejercicios clasificados en tres grupos (Grammatische Übungen, Hörverstehen, Übungen zum Leseverstehen) que tratan contenidos didácticos equivalentes al B2 del Marco de Referencia Europea.

Finalmente, las páginas sin diseño didáctico son aquellas que, si bien no están específicamente elaboradas para el aprendizaje del alemán como lengua extranjera, sí pueden ser explotadas para la práctica o adquisición de la misma. Contienen diccionarios, obras literarias, periódicos y revistas digitales, etc. En este apartado destacamos:

1 Wortschatz Lexikon (Universität Leipzig)

$<$ http://www.wortschatz.uni-leipzig.de/>: Esta página ofrece acceso a una base de datos donde se pueden consultar 35 millones de oraciones con 500 millones de términos del alemán actual.

1 Projekt Gutenberg-DE

$<$ http://gutenberg.spiegel.de/>: servidor que pone a disposición del usuario obras literarias desde hace diez años. Contiene más de 5.000 archivos de texto y de gráficos.

E-learning En este apartado, comentaremos nuesen la enseñanza tra experiencia como tutores telemátia distancia del cos de los cursos virtuales de alemán de alemán la Universidad Nacional de Educación a Distancia. Están implantados en la plalengua extranjera taforma de aprendizaje WebCT y están desarrollados siguiendo el modelo de cursos virtuales RPD (Rapid Prototyping Design), que se fundamen- ta en modelos pedagógicos formativos y cuya creación y perfeccionamiento se basa en repetidos procesos evaluativos. Los cursos se componen básicamente de cuatro herramientas para el aprendizaje, aunque trataremos únicamente el uso de las dos primeras:

1 Herramientas de contenido.

1 Herramientas de comunicación.

1 Herramientas para el estudio.

1 Herramientas para la evaluación.

El módulo de contenidos contiene el material didáctico en formato electrónico que el alumno debe estudiarse. Junto a textos introductorios con ejercicios de comprensión, incluye material de refuerzo en los capítulos dedicados a la gramática, a la ampliación de vocabulario, así como a la práctica de las diferentes destrezas con ejercicios variados, que incluyen textos con enlaces diversos, animaciones, referencias al material sonoro, etc. Para la elaboración de la mayoría de los ejercicios de refuerzo, se utilizaron los programas «Hot Potatoes» y "Macromedia Flash MX», entre otros.

Además de los contenidos didácticos, este módulo incluye un glosario y el programa de la asignatura, entre otros recursos.

El área de las comuII Jy

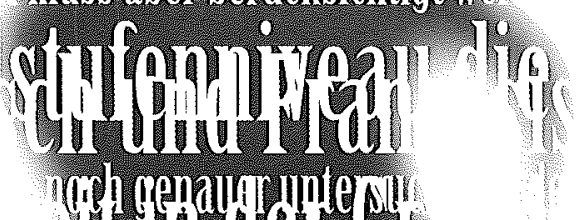
nicaciones en estos cursos se articula básicamente en torno a tres herramientas, dos de comunicación asíncrona el correo electrónico y los foros de debate - y una síncrona - la charla o chat. Nuestras investigaciones corroboran las opiniones generalizadas en el sentido de que en entornos de aprendizaje virtuales las funcionalidades de mayor relevancia corresponden, sin duda, a las comunicaciones. El buen funcionamiento de la comunicación entre la comunidad virtual es responsabilidad casi exclusiva del moderador de los diferentes foros o chats que se planifican con todo detenimiento. Podemos asegurar que existe un correcto desarrollo cuando se producen, entre otras, las siguientes circunstancias: participación eleva$\mathrm{da}$, negociaciones de significados, utilización por parte de los alumno de manera espontánea y progresiva de la lengua meta en sus intervenciones, integración de los alumnos en el grupo de trabajo, etc.

El siguiente ejemplo muestra un 
fragmento de un chat, durante el cual los alumnos «conversan» con el objetivo de iniciar su trabajo de grupo. En este caso, se trata de realizar un ejercicio tipo Webquest con el resultado final de una redacción sobre alguna ciudad alemana ${ }^{3}$ :

Begoña D. >>vale

Inmaculada C.>>Si ponla

Maria Herminia V.W. >> La idea es la siguiente: Leipzig ist eine Stadt im nordwestlichen Teil Sachsens. Es hat ca. 500.000 Einwohnern. Es ist sebr schön und hat viel Sehenswiirdigkeiten, Komfortable Unterkunft, Gutes Essen und ein Schöne Landschaft. Leipzig hat auch eine Messe und ist verkehrsgiinstig. Luego podemos desarrollar estos temas, como si se tratara de un folleto turístico.

Maria Herminia V.W. >>Cada una preparamos unos temas Inmaculada $C>>$ sabeis que extension tiene que tener el texto? Maria Herminia V.W. >SSólo 100 palabras $>$ Así que no hay que enrollarse mucho

Inmaculada C. >>Pues me parece bien $\gg$ Th idea,quiero decir

Begoña $D \gg$ y a mi

Inmaculada C. $>>$ Yo be becho una pequeña redaccion

Maria Herminia V.W. >>Pues si una prepara: Sehenswïrdigkei- ten un die Messe, otra Komfortable Unterkunft und Gutes Essen y otrs Schöne Landschaft und verkehrsgÑunstig

Maria Herminia V.W. >>Cual es tu radacción $>>$ Perdon ¿Cuál es tu redacción?

Inmaculada C.>>Es un poco simplepero me ayuda a poneme un poco en forma $>$ Quisiera saber que os parece esta frase: Leipzig ist die Bruckenfunktion zwischen Ost und West>ses hat viele Tradition und auch Innovation.Die Leipziger City ist liebens. wert mit ibren Kneipen und Cafes

Maria Herminia V.W. > bttp://hwuw.leipzig-sachsen.de/leipzig/tourismus.htm es un website que puede ayudarnos a encontrar información

A modo de conclusión, suscribimos plenamente las opiniones de Maragliano, que respondiendo a la pregunta « ¿El aprendizaje electrónico funciona de verdad? [...]» contestó

«Podría responder devolviéndole una pregunta similar: ¿La formación presencial funciona de verdad? [...] El problema es que ya no se puede prescindir de Internet [...] Por tanto, es impropio pensar; precisamente en el campo de la formación, en renunciar a este recurso, y es un lujo (que no nos podemos permitir) plantearnos dudas o perplejidades prejuiciosas al respecto».

\section{Bibliografia}

Bender T. (2003), Discussion Based Online

Teaching to Enbance Student Learning,

Virginia, Stylus Publishing.

Borrman, A. \& Gerdzen, R. (2000), Internet in Deutschunterricht, Stuttgart, Ernst Klett Verlag.

Breilmann, S. et al. (2003), Computer, Intemet o. Co. im Deutschunterricht ab Klasse 5, Berlin, Cornelsen.

Christensen, C. M. (1997), The Innovator's

Dilemma: When netw techologies cause great

firns to fail, Boston, Harvard Business School Press.

Cruz Piñol, M. (2002), Enseñar español en la era de Internet, Barcelona, Octaedro.

Döring, N. (1998), «Lernen und Lehren mit dem Internet» <http://www.nicola

doering.de/presentations/instruktion.htm>

[Consulta: 26.04.2005]

Ko, S. \& Rossen, S. (2001), Teaching Online: A Practical Guide, Boston, Houghton Mifflin Company.

Kolb, D.A. (1984), Experimental Learning:

Experience as the Source of Learning and

Development, Englewood Cliffs, N.J., Prentice Hall.

Maragliano, R. (2004): «Entrevista concedida a elearningeuropa,info».

\section{Notas}

$<h t$ tp:/elearningeuropa.info/index.php?page $=d$ oc\&doc_id $=5148 \&$ doclng $=7 \&$ menuzone $=3>$ [Consulta: 28.04.2005].

Rizek-Pfister, C. (2003): «Präsenzunterricht, Fernunterricht. Die Suche nach dem optimalen Mix", en Kerres, M. und Voß, B. (2003), Digitaler Campus. Vom Medienprojekt zum nachbaltigen Medieneinsatz in der Hochschule. Münster, Waxmann.

Rosenberg, M. J. (2001), E-Learning: Strategies for Delivering Knowledge in the Digital Age, New York, McGraw-Hill. Rossett, A. et al. (2003) «Strategies for Building Blended Learning» http://www.learningcircuits.org/2003/ jul2003/rossett.htm [Consulta: 27.04.2005] Sauter, A. M. et al. (2004), Blended Learning, München, Luchterhand.

Steinig, W. (2000): «Kommunikation im Internet: Perspektiven zwischen Deutsch als Erst-und Fremdsprache", en Zeitschrift fiir Fremdsprachenforschung, 11, (2).

Weller, M. (2002), Delivering learning on the Net, London, Kogan Page.
1. Universidad Virtual UOC

(http://www.uoc.edu), las iniciativas de universidades presenciales como la Complutense de Madrid y su campus virtual (https://campusvirtual1.ucm.es/cv/) o la enseñanza virtual que ofrece la UNED (http://www.uned.es) son tres buenos ejemplos de cada uno de estos modelos.

2. Sonja Gerber define de la siguiente manera el término Webquest «Der englische Begriff. 'Quest' steht für die ritterlichen

Abenteuerfahrten, wie sie in der Literatur des Mittelalters beschrieben werden, bedeutet im Englischen von heute aber auch eine anspruchsvolle Suche oder Nachforschung. 'WebQuest' steht für die Lösung von Aufgaben mit Hilfe von Informationen aus dem Internet»

$<$ http://www.englisch.schule.de/daf_webquest. htm\#konzept> [Consulta: 27.04.2005] Para más información sobre este tipo de ejercicios basados en modelos de aprendizaje constructivistas puede consultarse, entre otras, la dirección: <http://wwwwebquest.org> [Consulta: 27.04.2005]

3. Se trata de una reproducción literal del texto del chat sin corrección alguna por nuestra parte. 


\section{Studieren und forschen in Deutschland Estudiar e investigar en Alemania}

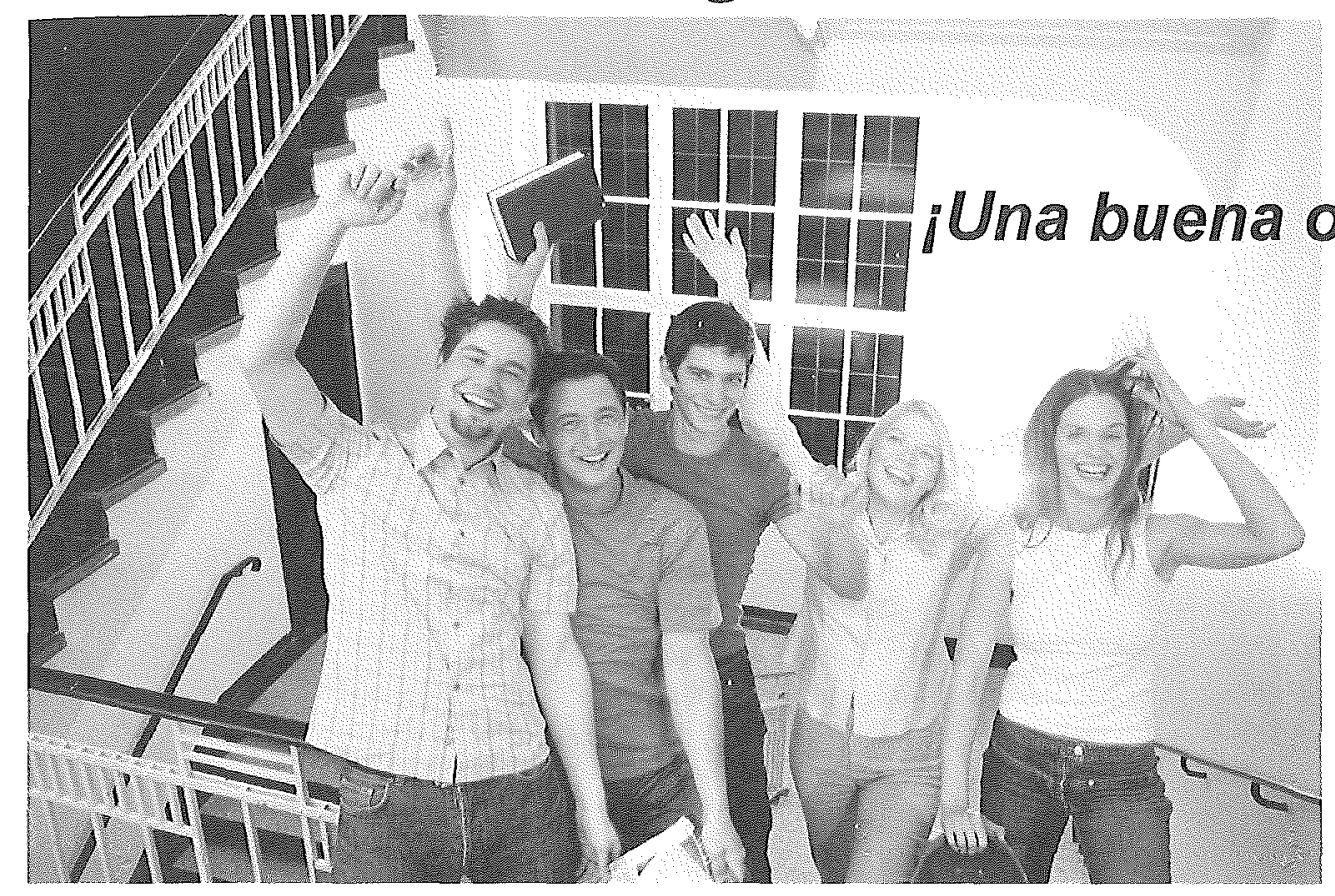

Seit Oktober 2004 informiert das neue DAAD-Informationszentrum (IC) in Barcelona über Studien- und Forschungsmöglichkeiten in Deutschland. Unsere Aufgaben:

- Beratung bei der Suche nach einem geeigneten Studiengang oder Hochschulort

- Information über Stipendien des DAAD und anderer Einrichtungen

- Hilfe bei der Bewerbung um ein Stipendium oder einen Studienplatz sowie bei der Vorbereitung des Aufenthaltes in Deutschland

- Unterstützung bei der Kontaktaufnahme mit deutschen Instituten und Professorinnen

- Durchführung von Vorträgen und Informationsveranstaltungen an Universitäten und Schulen

- Beteiligung an Hochschul- und Bildungsmessen in Spanien u.v.m.

Mit seiner Arbeit trägt das IC zur Bekanntmachung und Verbreitung der Angebote der deutschen Hochschulen und Forschungseinrichtungen in Spanien bei. Ausführliche Informationen und hilfreiche Links zum Studienstandort Deutschland finden Sie in deutscher und spanischer Sprache auf unserer Homepage:

\section{http:// ic.daad.de/barcelona}

DAAD Informationszentrum (IC)

C/. Manso, 24-28, $2^{\circ}$ (Goethe-Institut)

08015 Barcelona

TLf/Fax: (+34) 934244010

Email: daad@barcelona.goethe.org 